\title{
As características dos usuários do produto: uma ferramente para auxiliar na condução do envolvimento do usuário no PDP
}

Gustavo Michelini (gustavomichelini@gmail.com) - Escola de Engenharia de São Carlos, Universidade de São Paulo

João Vitor de Medeiros (jv.med96@gmail.com) - Escola de Engenharia de São Carlos, Universidade de São Paulo

Julia Inocenti di Giovanni (judigio01@gmail.com) - Escola de Engenharia de São Carlos, Universidade de São Paulo

Janaina Mascarenhas Hornos da Costa (janainamhcosta@gmail.com) - Escola de Engenharia de São Carlos, Universidade de São Paulo

\section{RESUMO}

O conhecimento sobre as características que os usuários devem conter para participar do desenvolvimento do produto, segundo Lettl (2007), é uma competência que a empresa precisa possuir para realizar o envolvimento efetivo dos usuários no PDP. Na literatura, existem diversos tipos de usuários desempenhando distintas funções no desenvolvimento do produto, o que dificulta o entendimento de qual usuário deve ser envolvido. Essa dificuldade é especialmente vista na indústria médica, na qual existe diferentes usuários, como médicos e técnicos de enfermagem, que utilizam tais produtos. Dessa forma, essa pesquisa buscou mapear as características que os usuários devem possuir para desempenhar essas distintas funções no PDP. Por exemplo, sendo o envolvimento do usuário do tipo "advanced user" ser recomendado para auxiliar na classificação e seleção das ideias, os usuários que forem desempenhar o papel de "classificar e selecionar ideias" deve ter a característica de possuir experiência de uso do produto. Como resultado, essa pesquisa identificou dez tipos e cinco características dos usuários. Esses resultados obtidos, apresentados nesse artigo, são resultados parciais de um projeto de pesquisa em andamento.

Palavras-chave: Envolvimento do usuário; tipos de usuários; pesquisa de marketing; PDP; gerenciamento de projetos

Área: Ferramenta e métodos de desenvolvimento de produtos e serviços.

\section{INTRODUÇÃO}

A recomendação de que o usuário deve ser envolvido no projeto de desenvolvimento não é algo novo na literatura e está coberta por diversas abordagens, entre elas: UCD - Usercentered Design (KUJALA, 2003), Marketing research (WEBER, 2008), Open innovation (CHESBROUGH, 2003), Contextual design (KUJALA, 2003), Participatory design (KUJALA, 2003), Cocreation (PRAHALAD; RAMASWAMY, 2004).

Von Hippel (1978) enfatizou que o usuário deveria ser envolvido no desenvolvimento quando constatou que a participação deles na identificação de oportunidades de novos produtos poderia agregar valor no desenvolvimento do produto. Assim, usuários, provenientes dos diferentes segmentos de mercado, podem ser envolvidos para avaliar os conceitos de produtos gerados (ULRICH; EPPINGER, 2012). 
Atualmente, constata-se uma evolução no conceito do que é envolver o usuário no desenvolvimento do produto. Os usuários ganharam novas funções no processo de desenvolvimento. $\mathrm{Na}$ abordagem Cocreation, por exemplo, é sugerido que as empresas devem interagir constantemente com os usuários, de maneira que sejam completamente ativos no projeto, chegando ao ponto de participar inclusive do design técnico e detalhado do produto (PRAHALAD; RAMASWAMY, 2004). Sendo assim, o usuário pode desempenhar diversas funções no PDP.

As funções que os usuários podem desempenhar estão dispersos nas diversas atividades que ocorrem no PDP, entre elas: fornecer ideias e novas oportunidades (COOPER, 2001; CRAWFORD; DI BENEDETTO, 2006), classificar e selecionar ideias (COOPER, 2001), fornecer conceitos de produtos (CRAWFORD; DI BENEDETTO, 2006), relatar suas necessidades (ULRICH; EPPINGER, 2012), avaliar conceitos de produto (ULRICH; EPPINGER, 2012), validar conceitos de produto (COOPER, 2001), avaliar protótipos (COOPER, 2001), testar produto em ambiente de uso (CRAWFORD; DI BENEDETTO, 2006) e participar de simulações de vendas (CRAWFORD; DI BENEDETTO, 2006).

As distintas funções do PDP exigem diferentes características dos usuários participantes. Pesquisas apontam que diferentes características dos usuários, do ponto de vista do indíviduo, são requisitadas para desempenhar tais funções (LETTL, 2007). Assim, um único usuário não é suficiente para contemplar todo o projeto (WEBER, 2008). Por exemplo, os autores Kristensson et al. (2004) explicam, em seu estudo sobre sistemas de telefonia móvel, que usuários do tipo "ordinary users", caracterizados por não possuírem muita experiência e habilidade técnica com o produto, estão mais aptos a gerar ideias originais, enquanto que "professional users" e "advanced users", por terem a característica de possuírem conhecimento sobre o funcionamento e tecnologia do produto, podem auxiliar na classificação e seleção das ideias.

Para Lettl (2007), conhecer as características dos usuários é uma competência que a empresa precisa possuir para realizar um envolvimento efetivo do usuário no desenvolvimento. $\mathrm{O}$ autor sugere que duas competências são necessárias. A primeira competência está relacionada com o conhecimento sobre os usuários, sobre como os diferentes tipos de usuários podem participar do processo de desenvolvimento. A segunda competência está relacionada com o padrão de interação entre a empresa e o usuário, representados pela quantidade de usuários, tempo e forma de interação entre usuário e a empresa. Dessa forma, é importante a empresa ter o conhecimento dos usuários certos a serem envolvidos no desenvolvimento.

A literatura aborda superficialmente quais usuários devem ser envolvidos no desenvolvimento, enfatizando apenas quais usuários, ou público, o novo produto deve atender. Porém, é válido enfatizar que a definição de quem são os diferentes usuários de um produto deve ser o ponto de partida do projeto. Na indústria médica, por exemplo, um único produto possui vários usuários: o médico que utiliza o resultado de um exame, a técnica de enfermagem que irá de fato operar o produto, o paciente que interage de forma pacífica com o produto, usuários do final de vida do produto que irão fazer o descarte etc. Esses diferentes usuários deveriam ser envolvidos, ao longo do projeto, desempenhando funções distintas. Por exemplo, a técnica de enfermagem poderia ser envolvida para realizar testes de usabilidade, pois é a pessoa que de fato irá utilizar, ou operacionar, o produto. Nesse caso, não cabe ao médico ser a única pessoa a realizar a função de avaliar protótipos.

De fato, envolver o usuário é uma atividade desafiadora para as empresas. Quem são os usuários mais apropriados para serem envolvidos no desenvolvimento do produto? Essa é a pergunta-chave que as empresas encontram dificuldades no início do projeto. Posto isto, o objetivo desse trabalho é apresentar um levantamento das características que devem ser 
levados em consideração no processo de seleção dos usuários a serem envolvidos no desenvolvimento. A seguir, é discorrido sobre os conceitos teóricos dessa pesquisa, as etapas de pesquisa e, por fim, o resultado do trabalho e a discussão são reportadas.

\section{ENVOLVIMENTO DO USUÁRIO NO DESENVOLVIMENTO DO PRODUTO}

Para a autora Kujala (2008), o envolvimento do usuário no desenvolvimento de produtos é descrito como o contato direto da equipe de desenvolvimento com o usuário. A autoria define que o envolvimento do usuário é um conceito vago e coberto por diversas abordagens teóricas, os quais ela destaca: Participatory Design, estudos etnográficos, Design Centrado no Usuário e estudos de contexto de uso do produto. Na prática, o envolvimento pode ser realizado de forma ativa ou passiva, sendo a principal diferença entre elas o quão ativo é o papel do usuário no projeto. A participação ativa do usuário é definida pelo quanto ele influencia nas decisões e se ele participa como membro da equipe do projeto de desenvolvimento (KUJALA, 2008).

Alguns autores propuseram modelos que tentam explicar o envolvimento do usuário no desenvolvimento do produto. Von Hippel (1978) propôs a existência de dois paradigmas quanto ao envolvimento do usuário no processo de geração de ideias no desenvolvimento de produtos. O primeiro paradigma, chamado de Manufacturer-Active Paradigm - MAP, é caracterizado pelo fato de o usuário fornecer suas necessidades e desejos à empresa desenvolvedora do produto, enquanto que o segundo paradigma, chamado de CustomerActive Paradigm - CAP, se caracteriza pelo fato de o usuário possuir uma ideia de produto e, assim, selecionar uma empresa manufatureira para fornecer sua ideia. No CAP, o papel da empresa é julgar tal ideia para decidir se irá ser produzida ou não. Em contrapartida, Foxall (1984) diz que o comportamento empreendedor citado por Von Hippel no CAP é desempenhado pela empresa. Assim, Foxall sugere a existência de um terceiro paradigma, o CAP2, caracterizado pelo comportamento empreendedor do usuário, o qual é capaz de patentear suas ideias para obter ganhos com a comercialização do produto.

Weber (2008) declarou que desenvolver produtos e serviços sem considerar as necessidades dos usuários levará ao fracasso do projeto, gerando custos extras. O autor também afirma que os custos do projeto podem ser reduzidos quando os usuários são envolvidos pois, de alguma forma, eles garantem a qualidade do novo produto. As empresas que envolvem acreditam que os usuários são experts quanto ao domínio do conhecimento das atividades, práticas, comportamentos e preferências e, assim, são representantes do contexto geral de uso do produto (KUJALA, 2008).

As empresas enfrentam diversos desafios quanto à condução do envolvimento do usuário no desenvolvimento. Uma grande dificuldade está relacionada com a seleção dos métodos, que devem ser utilizados em cada uma das fase do projeto, para apoiar o envolvimento do usuário (COOPER; DREHER, 2010). Uma outra dificuldade que as empresas enfrentam é escolher os usuários corretos a serem envolvidos nas diferentes fases do projeto (LETTL, 2007). Para casos de inovação radical, por exemplo, saber quem são os usuários mais apropriados a serem envolvidos na fase certa do projeto é crucial, pois decisões do projeto podem afetar o sucesso do novo produto (LETTL, 2007).

As duas competências, que a empresa precisa possuir para envolver o usuário, propostas por Lettl (2007) e ilustrada na Figura 1, estão relacionadas com duas dimensões: a dimensão do usuário e a dimensão da interação entre usuário e a empresa. A primeira refere-se ao conhecimento sobre quais são as características críticas dos usuários e como os diferentes tipos de usuários podem contribuir nas diferentes fases do projeto. A segunda dimensão 
refere-se ao processo de interação que a empresa terá com o usuário, sendo definido como o número de usuários envolvidos, tempo de interação e forma de interação.

Figura 1. Competências para envolver o usuário. Fonte: Lettl (2007)

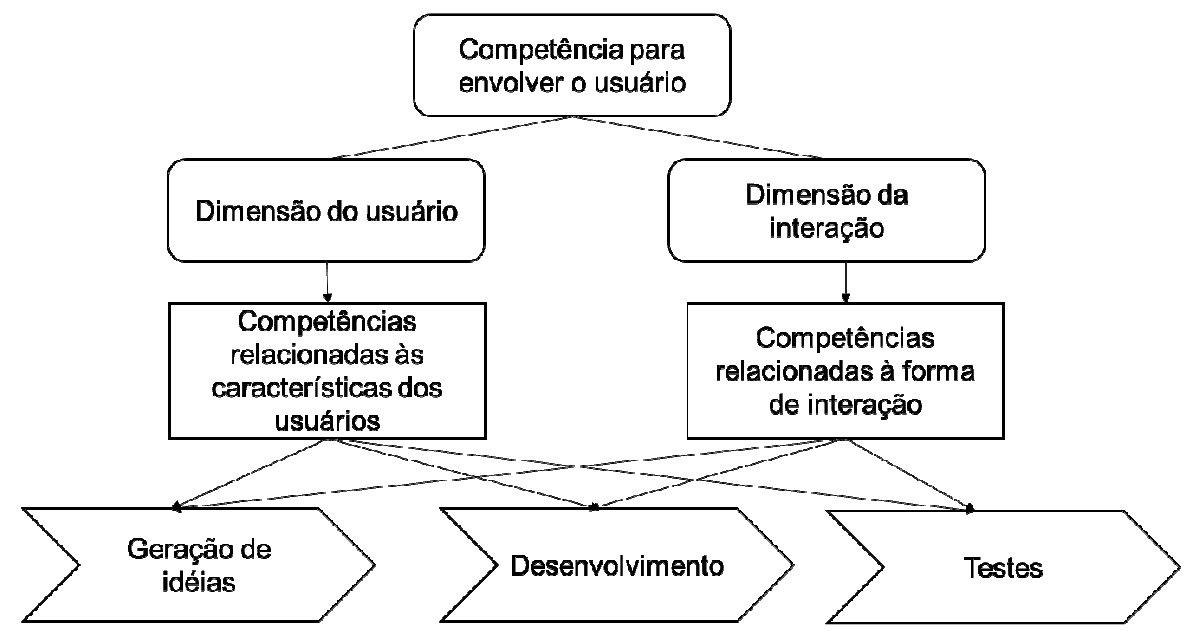

Além de definir qual usuário deve ser envolvido, e a forma de interação adequada, a empresa deve decidir o nível de envolvimento. Olsson (2004) sugere que o nível de envolvimento do usuário seja definido pelo quão ativo é a participação do usuário no projeto. Para o autor, o usuário pode ser visto apenas como um sujeito e, portanto, seu envolvimento ser passivo, e sua pessoa representada através de Personas, ou pode até ser visto como um parceiro no projeto, atuando como membro da equipe de desenvolvimento. Os níveis de envolvimento propostos pelo autor estão ilustrados na Figura 2.

Figura 2. Níveis de envolvimento do usuário. Fonte: Olsson (2004)

\section{Usuários como parceiros Usuários como informantes Usuários como sujeitos}

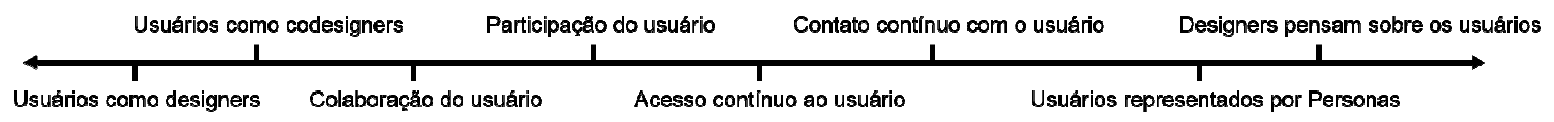

Desse modo, o nível de envolvimento está diretamente relacionado com o seu papel do usuário no processo de desenvolvimento. Kaulio (1998) propôs que o envolvimento possui três níveis. No primeiro nível, "design for", a interação com o usuário é caracterizada pela busca e coleta de dados em relação ao consumidor através de teorias gerais e modelos comportamentais. No segundo nível, "design with", o envolvimento é caracterizado pela avaliação de diferentes soluções e conceitos de produto, para que se entenda as preferências dos consumidores. Por fim, no terceiro nível, "design by", há participação ativa do usuário no desenvolvimento, sendo possivelmente um membro ativo na equipe de projeto.

Dessa forma, como diferentes fases do projeto exigem diferentes habilidades e conhecimentos do usuário a ser envolvido (WEBER, 2008), as empresas precisam saber quais tipos de usuários, definidos pelas características que possuem, são capazes de contribuir nas diferentes fases do projeto (LETTL, 2007).

Na literatura são propostas algumas definições de tipos e características de usuários. Von Hippel (1978) propôs a definição do tipo de usuário "lead users". Twedt (1964) discutiu a importância de se entender quem são os "heavy users", "light users" e "non-users" de um produto. Kristensson et al. (2004), investigando a criatividade das ideias propostas por usuários, propôs outros quatro tipos de usuários: "ordinary users", "developer users", "professional users" e "advanced users". Lettl (2007), por sua vez, identificou mais dois tipos: "inventive users" e "extreme users". 
As propostas desses tipos de usuários foram realizadas sob diferentes contextos, variando principalmente a indústria e o tipo de projeto. No entanto, elas são válidas para esse trabalho pois expõem as diferentes maneiras de entender quem são tipos de usuários e as características que os distinguem. Ressalta-se que as diferentes fases do projeto de produto requerem diferentes características dos usuários participantes, implicando que um único tipo de usuário não é suficiente para contemplar todo o projeto (WEBER, 2008).

\section{METODOLOGIA}

Com o propósito de entender quais são as características que os usuários devem possuir para desempenhar as distintas funções no PDP, a pesquisa foi dividida em duas fases. A primeira fase teve por objetivo consolidar os tipos de usuário presentes na literatura. Foi realizada uma revisão sistemática com o objetivo específico de identificar artigos que apresentassem tipos de usuários de um produto. A busca foi realizada na base de dado da Web of Science, utilizando as palavras-chave "user involvement" e "product development", unidos por meio do operador lógico AND. De um total de 91 artigos, após a leitura dos resumos, foi indicado a leitura integral dos seguintes textos: Damodaran (1996), Grudin (1991), Holt (1988), Hoyer et al. (2010), Kaulio (1998), Kristensson et al. (2004), Kujala (2003), Kujala (2008), Lettl (2007), Prahalad \& Ramaswamy (2004), von Hippel (1978), Von Hippel et al. (2011), Weber (2008) e Wilkinson; De Angeli (2014). Esses artigos foram, portanto, também utilizados como base no mapeamento dos tipos de usuários.

A segunda fase, após a identificação dos tipos dos usuários, teve como objetivo a identificação das características dos usuárias contidas de forma direta e indireta nos tipos de usuários. Assim, foi feita uma análise criteriosa para extrair o que caracteriza cada tipo de usuário e, com isso, coletadas as diferentes características intrínsecas dos usuários de um produto.

$\mathrm{Na}$ realização desse mapeamento inicial dos tipos e características dos usuários, foram utilizadas as seguintes definições:

- Tipos de usuário: grupo de consumidores com característica(s) semelhante(s). Por exemplo, "heavy user" é um tipo de usuário com a característica de alta frequência de uso, ou compra, do produto;

- Características dos usuários: algo que distingue um usuário de outro. Nessa pesquisa, somente características ligadas ao uso do produto são consideradas. Por exemplo, a frequência que usa o produto e o conhecimento tecnológico que possui do produto são características que distinguem os usuários, caracterizando, respectivamente, os usuários do tipo "heavy user" e do tipo "advanced user".

\section{RESULTADOS}

Foram identificados, no mapeamento, dez tipos de usuários na literatura: Heavy user, Light user e Non user Ordinary user, Lead user, Advanced user, Developer user, Professional user, Inventive user e Extreme user (Quadro 1).

Twedt (1964) identificou três tipos de usuários: Heavy user, Light User e Non User. Preocupado com a segmentação de mercado, o autor realizou análises sobre as concentrações de compras de 700 casas de famílias em 18 categorias de produto. Assim, o autor constatou que uma parcela de famílias, as quais chamou de "heavy users", possuíam um volume de compras nove vezes maior que o volume das outras famílias que também compravam o produuto, chamadas de "light users". Twedt também definiu que "non user" representa a 
situação em que a família não comprava uma categoria de produto. Para verificar a consistência dessa tipologia, os autores Cook \& Mindak (1984) analisaram o consumo e o intervalo de uso de determinadas categorias de produto em uma base de dados nacional, enquanto que a análise de Twedt foi relatada apenas em uma cidade. Mesmo assim, os autores Cook \& Mindak (1984) validaram a existência desses três tipos de usuários relacionados com a frequência que compram ou usam o produto.

Von Hippel (1986) declarou que os usuários do tipo "lead users" são as pessoas mais acuradas para a geração de novos conceitos. Para o autor, "lead users" estão familiarizados com as condições consideradas futuro para outras pessoas. Assim, o autor definiu que o "lead user" de um novo produto possui duas características: 1) possuem necessidades, no momento, que serão desejadas no futuro pelas outras pessoas e 2) serão altamente beneficiados com a solução de tal necessidade. Dessa forma, o autor define que os "lead users" podem participar do projeto de inovação por meio do fornecimento de suas necessidades. A teoria de que usuários do tipo "lead users" podem ser utilizados em casos de inovação também é aconselhada por outros autores como Kaulio (1998), Lettl, (2007) e Weber (2008).

Kristensson et al. (2004), por meio de um estudo no serviço de tecnologias móveis, analisou a criatividade das ideias fornecidas por quatro tipos de usuários distintos: Professional user, Developer user, Advanced user e Ordinary user. O autor definiu "professional users" como indivíduos que possuem experiência com o uso do produto, enquanto que "developer users" já participam do desenvolvimento do produto em questão, "advanced users" possuem conhecimento técnico, como um curso sobre o uso do produto, e "ordinary users" são indivíduos que não possuem conhecimento profissional com o produto. Em seu estudo, o autor constatou que "ordinary users" geram ideias divergentes, mais originais que os outros usuários, mas que havia pouca chances de serem realizáveis, devido às restrições da empresa e da tecnologia. Os usuários dos tipos "professional users", "advanced users" e "developer users", por terem conhecimento prévio sobre o funcionamento, desenvolvimento e tecnologia de tal produto, apresentavam soluções convergentes e realizáveis pela empresa, mas pouco originais.

Lettl (2007), por meio de um estudo de casos múltiplos em produtos médicos, apresentou outros tipos de usuários: Inventive users e Extreme users. Em seu estudo, o autor definiu "inventive users" como médicos que realizavam modificações ou criavam novos conceitos de produtos e "extreme users" como médicos que possuíam necessidades especiais. Assim, o autor observou que "inventive users" poderiam fornecer protótipos para casos de inovação, pois eles antecipam as novas tecnologias da indústria médica. Os "extreme users", nos casos relatados pelo autor, auxiliaram a equipe na definição das necessidades de maneira mais acurada. Por exemplo, alguns neurocirurgiões precisam de instrumentos com extrema precisão e necessitam que o novo produto os atendam.

Quadro 1. Tipos de usuários compilados. Fonte: autor

\begin{tabular}{|c|c|c|c|}
\hline $\begin{array}{c}\text { Tipologia proposta } \\
\text { por Twedt (1964) }\end{array}$ & $\begin{array}{c}\text { Tipologia proposta } \\
\text { por Von Hippel } \\
(1986)\end{array}$ & $\begin{array}{c}\text { Tipologia proposta por } \\
\text { Kristensson et al. (2004) }\end{array}$ & $\begin{array}{c}\text { Tipologia proposta } \\
\text { por Lettl (2007) }\end{array}$ \\
\hline 1. Heavy user & 4. Lead user & $\begin{array}{l}\text { 5. Advanced user } \\
\text { 6. Developer user }\end{array}$ & 9. Inventive user \\
2. Light user & & $\begin{array}{l}\text { 7. Professional user } \\
\text { 3. Non user }\end{array}$ & 10. Extreme user \\
\hline
\end{tabular}


A partir dos tipos de usuários listados, foi possível identificar as características dos usuários relacionadas com o uso do produto. Nesse identificação, foi extraído o que caracteriza cada tipo de usuário. Por exemplo, "heavy user" possui a característica de utilizar o produto com muita frequência. Assim, a partir do tipo de usuário "heavy user", extraiu-se a característa de "frequência de uso". Portanto, foram mapeadas as características intrínsecas que os usuários devem possuir para desempenhar as distintas funções no PDP.

O resultado dessa análise são cinco características dos usuários:

Frequência de uso. A característica de frequência de uso está relacionada, nesse trabalho, com os usuários dos tipos "heavy users", "light users" e "non user". Segundo o autor Twedt (1964), a frequência com que certos usuários compram, ou usam, um produto é uma característica importante para que possam participar do desenvolvimento fornecendo suas necessidades e avaliando conceitos de produtos.

Experiência com o uso. A experiência de uso do produto está relacionado com os usuários dos tipos "lead users", "advanced users" e "professional users". Usuários com alta experiência, chamados de "professional user", possuem melhor condições de desempenhar os papéis de avaliar protótipos e testar em ambiente de uso (KRISTENSSON et al., 2004). Usuários que possuem experiência com o uso, e serão altamente beneficiados com o projeto de novo produto inovador, são chamados de "lead user" e podem participar da geração de conceitos (VON HIPPEL, 1986).

Conhecimento tecnológico. O conhecimento sobre a tecnologia do produto é uma característica dos "advanced user". Para Kristensson et al. (2004), esses indivíduos podem classificar e selecionar ideias, já que possuem conhecimento sobre a tecnologia que o novo produto necessita.

Necessidades extremas. As necessidades especiais caracterizam os usuários dos tipos "lead user" e "extreme user". Usuários que possuem necessidades extremas, chamados por Lettl (2007) de "extreme user", requerem características do produto que a maioria dos usuários não necessitam e, por isso, podem desempenhar o papel de relatar suas necessidades.

Habilidade de pesquisa. Os usuários com habilidades para pesquisar apresentam características de modificarem o produto, ou de desenvolverem novos conceitos e tecnologias. Eles são chamados de "inventive user" por Lettl (2007). Segundo o autor, esses usuários podem auxiliar na condução de projetos de inovação radical por meio do fornecimento de novas ideias e conceitos para a equipe de projeto.

\section{DISCUSSÃO}

Embora conhecer as diferentes características dos usuários seja uma competência que a empresa precisa possuir para envolver o usuário no desenvolvimento do produto (LETTL, 2007), frequentemente as empresas consideram os usuários como um grupo homogêneo (SCHWEITZER et al., 2014). Como diferentes fases do projeto de produto requerem diferentes características dos usuários participantes (WEBER, 2008), as empresas precisam ter conhecimento das diferentes características que os usuários devem possuir para desempenhar as distintas funções no PDP.

Nesse artigo, são listados dez tipos de usuários distintos: heavy user, light user, non user, ordinary user, lead user, advanced user, developer user, professional user, inventive user e extreme user. A partir da coleta desses tipos de usuários, cinco características dos usuários, relacionadas com o uso do produto, foram listadas: frequência de uso do produto, experiência com o uso, conhecimento tecnológico, necessidades extremas e habilidades de pesquisa. 
Dessa forma, esses resultados demonstram que existem diferentes tipos de usuários e que esses diferentes tipos podem contribuir de formas distintas, por meio de diferentes funções, no desenvolvimento do produto. Como constatado por Lettl (2007), os diferentes usuários, por possuírem características distintas, podem contribuir de maneiras diferentes no projeto de novo produto.

\section{CONSIDERAÇÕES FINAIS}

Essa pesquisa teve como objetivo a realização de um primeiro mapeamento das características que os usuários devem possuir para desempenhar as distintas funções no PDP. Para isso, foi realizado uma revisão na literatura de PDP e na literatura de envolvimento do usuário. Foram coletados dez tipos e cinco características dos usuários.

Portanto, embora frequentemente as empresas lidem com os usuários como se fosse um grupo homogêneo de pessoas, o resultado desse artigo demonstrou que existem diversos tipos de usuários de um produto. Entre os tipos de usuários mapeados, podemos listar alguns como "heavy user" e "inventive user". A diferença entre os tipos de usuários acontece por meio das dististas características que os distinguem. Por exemplo, os usuários que possuem a característica de alta frequência de uso do produto são chamados de "heavy user" e os usuários com característica de possuírem habilidades de pesquisa são chamados de "inventive user". Assim, pode-se constatar na teoria que diferentes tipos de usuários, por possuírem diferentes características, devem desempenhar funções distintas no PDP.

\section{REFERÊNCIAS}

CHESBROUGH, H. W. The era of Open Innovation. MIT Sloan Management Review, p. 35-42, 2003.

COOK, V. J. J.; MINDAK, W. A. A Search for Constants: The "Heavy User" Revisited! Journal of Consumer Marketing, v. 1, n. 4, p. 79-81, 1984.

COOPER, R. G. Winning at New Products: Accelerating the Process from Idea to Launch. New York: Perseus Publishing, 2001.

COOPER, R. G.; DREHER, A. Voice-of-customer methods: What is the best source of newproduct ideas? Marketing Management Magazine, n. Winter, p. 38-48, 2010.

CRAWFORD, M.; DI BENEDETTO, A. New Products Management. New York: McGrawHill, 2006.

DAMODARAN, L. User involvement in the systems design process-a practical guide for users. Behaviour \& Information Technology, v. 15, n. 6, p. 363-377, 1996.

FOXALL, G.; TIERNEY, J. From CAP1 to CAP2: User-initiated innovation from the user's point of view. Management Decision, v. 22, n. 5, p. 3-15, 1984.

GRUDIN, J. Obstacles to user involvement in software product development, with implications for CSCW. International Journal of Man-Machine Studies, v. 34, n. 3, p. 435-452, 1991.

HOLT, K. The role of the user in product innovation. Technovation, v. 7, n. 3, p. 249-258, 1988.

HOYER, W. D. et al. Consumer Cocreation in New Product Development. Journal of Service Research, v. 13, n. 3, p. 283-296, 2010. 
KAULIO, M. A. Customer, consumer and user involvement in product development: A framework and a review of selected methods. Total Quality Management, n. 1358, p. 141149, 1998.

KRISTENSSON, P.; GUSTAFSSON, A.; ARCHER, T. Harnessing the creative potential among users. Journal of Product Innovation Management, v. 21, n. 1, p. 4-14, 2004.

KUJALA, S. User involvement: a review of the bene ${ }^{\circledR}$ ts and challenges. Behaviour Information Technology, v. 22, n. 1, p. 1-16, 2003.

KUJALA, S. Effective user involvement in product development by improving the analysis of user needs. Behaviour \& Information Technology, v. 27, n. 6, p. 457-473, 2008.

LETTL, C. User involvement competence for radical innovation. Journal of Engineering and Technology Management - JET-M, v. 24, n. 1-2, p. 53-75, 2007.

OLSSON, E. What active users and designers contribute in the design process. Interacting with Computers, v. 16, n. 2, p. 377-401, 2004.

PRAHALAD, C. K.; RAMASWAMY, V. co-Creating Unique Value With Customes. Strategy and Leadership, v. 32, n. 3, p. 32,3, 2004.

SCHWEITZER, F.; GASSMANN, O.; RAU, C. Lessons from ideation: Where does user involvement lead us? Creativity and Innovation Management, v. 23, n. 2, p. 155-167, 2014.

TWEDT, D. W. How Important to Marketing Strategy Is the "Heavy User"? Journal of Marketing, v. 28, n. 1, p. 71-72, 1964.

UlRICH, K. T.; EPPINGER, S. D. Product Design and Development. New York: McGraw-Hill, 2012.

VON HIPPEL, E. Successful Industrial Products from Customer IdeasJournal of Marketing, 1978. Disponível em: 〈http://www.jstor.org/stable/1250327?origin=crossref>

VON HIPPEL, E. Lead Users: A Source of Novel Product Concepts. Management Science, v. 32, n. 7, p. 791-805, 1986.

VON HIPPEL, E.; OGAWA, S.; JONG, J. P. J. The age of the consumer-innovator. MIT Sloan Management Review, v. 53, n. 1, p. 27-35, 2011.

WEBER, M. Developing what customers really need: Involving customers in innovations. Proceedings of the 4th IEEE International Conference on Management of Innovation and Technology, ICMIT. Anais...2008

WILKINSON, C. R.; DE ANGELI, A. Applying user centred and participatory design approaches to commercial product development. Design Studies, v. 35, n. 6, p. 614-631, 2014. 